\title{
Primer Estudio de Investigación de la Aplicación del Protocolo Grupal e Integrativo con EMDR a Niños Víctimas de Violencia Interpersonal Severa
}

\author{
Ignacio Jarero \\ Susana Roque-López \\ Julio Gómez \\ Fundación Latinoamericana y del Caribe para la Investigación del Trauma Psicológico. \\ Latin American \& Caribbean Foundation for Psychological Trauma Research. \\ Innocence in Danger-Colombia.
}

\begin{abstract}
El presente estudio, evalúo un abordaje de tratamiento del trauma, multi-componente y en fases, aplicado a 34 niñas y niños que fueron víctimas de violencia interpersonal severa (e.g., violación, abuso sexual, violencia física y emocional, negligencia y abandono). Los niños asistieron a un campamento de recuperación del trauma que tuvo una duración de una semana, en el que se les facilitaron experiencias para desarrollar recursos psicológicos, y en el que se les administró el Protocolo Grupal e Integrativo con Terapia de Reprocesamiento y Desensibilización a través del Movimiento Ocular (EMDR-IGTP) y terapia individual de EMDR, para la resolución de las memorias traumáticas. Las sesiones de terapia individual de EMDR, se administraron a 26 niños y niñas que aún presentaban perturbación relacionada con las memorias traumáticas elegidas como blanco, después de la administración del Protocolo Grupal e Integrativo con EMDR. Los resultados mostraron una mejoría significativa en la Escala Child's Reaction to Traumatic Events Scale (CRTES) y en el Short PTSD Rating Interview (SPRINT) para todos los participantes. Estos resultados se mantuvieron en el seguimiento. Es necesario realizar más investigación para evaluar el Protocolo Grupal e Integrativo con EMDR y la terapia individual de EMDR, como parte de una aproximación terapéutica multi-modal para el tratamiento de niños que han sufrido violencia interpersonal severa.
\end{abstract}

Palabras clave: Protocolo Grupal e Integrativo con EMDR; EMDR con niños, trauma complejo, trauma interpersonal en niños, terapia multi-componente y en fases.

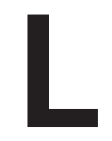
a violencia infantil es un fenómeno multidimensional que debe ser atendido con respuestas diversas. Proteger a las niñas y niños contra la violencia es un asunto urgente. Durante siglos han sufrido de violencia por parte de los adultos que ha pasado desapercibida. Los niños y niñas deben ser provistos de una prevención y protección efectiva de aquello que representa sus derechos inalienables (Naciones Unidas, 2006).

La violencia interpersonal, ya sea experimentada de forma directa o indirecta, especialmente durante la niñez, puede precipitar el Trastorno por Estrés Postraumático (TEPT) y/o ser un factor de riesgo que incremente la posibilidad de desarrollarlo ante traumas subsecuentes (Brewing, Andrews, \& Valentine, 2000).

Los niños y niñas cuyos traumas ocurren en un contexto de relaciones interpersonales, desarrollan síntomas asociados al TEPT (American Psychiatric Association [APA], 2000), tales como, dificultad para confiar, problemas en la regulación de sus emociones, somáticos, en el control de impulsos y en la identidad.

This is a modified translation of an article originally published as Jarero, I., Roque-López, S., Gómez, J. (2013). The Provision of an EMDR-Based Multicomponent Trauma Treatment with Child Victims of Severe Interpersonal Trauma. Journal of EMDR Practice and Research, 7(1), 17-28. Translated by Ignacio Jarero. 
Aquellos que han sufrido de abuso, comúnmente desarrollan síntomas adicionales relacionados con la auto-eficacia y la sexualidad (Van der Kolk, 2002).

Un maltrato excesivo en la infancia, se asocia con diversos efectos biológicos que alteran el desarrollo neurológico (De Bellis \& Van Dillen, 2005). Esta vulnerabilidad biológica genera déficits en el procesamiento de las emociones y en el funcionamiento ejecutivo, que lleva a una auto-regulación deficiente y a trastornos psiquiátricos posteriores, tales como TEPT, depresión y otros problemas emocionales (Van der Kolk, 2005). La investigación sobre Trastorno por Estrés Postraumático entre jóvenes que son objetos de maltrato ha prosperado debido a la importante prevalencia del trastorno entre este grupo (Pecora, White, Jackson, \& Wiggins, 2009).

\section{Tratamiento de Niños que han sufrido abuso}

Una aproximación multi-componente y en fases se recomienda ampliamente para el tratamiento del estrés traumático complejo (e.g. Courtois \&Ford, 2009). La primera fase de tratamiento se focaliza en la seguridad del paciente, en la estabilización de los síntomas y en el mejoramiento de sus competencias básicas de vida. La segunda fase, incluye la exploración de las memorias traumáticas, reduciendo primero la perturbación emocional aguda causada por dichas memorias, y posteriormente re-evaluando su significado para integrarlas en una identidad positiva y coherente.

La International Society for Traumatic Stress Studies (ISTSS), condujo una encuesta entre clínicos expertos, sobre las mejores prácticas para tratar el Trastorno por Estrés Postraumático (TEPT) Complejo (Cloitre et al., 2011). Las aproximaciones para la primera fase de tratamiento que recibieron la más alta calificación, fueron las estrategias focalizadas en las emociones y para la regulación de las mismas; mientras que, la educación acerca del trauma y la Atención Plena (mindfulness), obtuvieron el segundo lugar. Dentro de las aproximaciones para la segunda fase de tratamiento, la terapia individual fue identificada como de primera línea para el procesamiento de las memorias traumáticas y la conjunción del trabajo grupal con terapia individual, obtuvo el segundo lugar.

\section{La Terapia de Reprocesamiento y Desensibilización a través del Movimiento Ocular}

La Terapia de Reprocesamiento y Desensibilización a través del Movimiento Ocular (EMDR, por sus siglas en inglés), es recomendada para el tratamiento del Trastorno por Estrés Postraumático, tanto en adultos como en niños, por numerosas directrices internacionales como la Cochrane Review (Bisson \& Andrew, 2007) y el National Collaborating Centre for Mental Health (2005). Existe también evidencia preliminar que apoya su aplicación en el tratamiento de otros trastornos psiquiátricos, para varios problemas de salud mental y síntomas somáticos.

El marco teórico en el que se basa la Terapia EMDR, es el Modelo del Sistema de Procesamiento de Información (Adaptive Information Processing [AIP] Model). Shapiro (2001), plantea que gran parte de la psicopatología, es causada por una codificación inadecuada, del procesamiento incompleto de experiencias de vida traumáticas o perturbadoramente adversas.

Se piensa que esto afecta la habilidad del individuo para integrar estas experiencias de forma adaptativa. Se dice que la Terapia EMDR, la cual consiste en un proceso de 8 fases y tres etapas, facilita la reanudación del procesamiento normal de información y la integración de la misma.

Esta aproximación terapéutica que se enfoca en la experiencia pasada, los detonantes actuales y los retos potenciales a futuro, puede frecuentemente dar como resultado, el alivio de los síntomas presentes, con la disminución o desaparición de la perturbación relacionada a las memorias traumáticas; una imagen más positiva de sí mismo, el alivio de las molestias corporales, y la resolución de detonantes presentes, o los que se puedan presentar en el futuro.

El esclarecimiento y evolución de los mecanismos neurobiológicos (desconocidos para cualquier forma de psicoterapia) y de los modelos teóricos, está avanzando a través de la investigación y el desarrollo de la teoría (Asociación Internacional de EMDR [EMDRIA, por sus siglas en inglés], 2011).

Existe un cuerpo de investigaciones que evalúan el tratamiento con la Terapia EMDR aplicada a niños con traumas Tipo 1 (incidente único): desastres naturales (Chemtob, Nakashima, \& Carlson, 2002; Fernández, 2007; Greenwald, 1994), asalto (Coco \& Sharpe, 1993) y accidentes automovilísticos (Kemp, Drummond, \& McDermott, 2010; Ribchester, Yule, \& Duncan, 2010). Se ha encontrado que la Terapia EMDR en su modalidad individual, ha sido muy efectiva para reducir los síntomas del estrés postraumático en niños que sufrieron algún trauma. Revisar Adler-Tapia \& Settle (2008) y Fleming (2012).

Existen pocos estudios, que han investigado específicamente el tratamiento con terapia EMDR, en niños con traumas Tipo 2 (experiencias duraderas, como abuso sexual o guerra; Fleming, 2012). 
Un ensayo aleatorio controlado, realizado por Jaberghaderi, Greenwald, Rubin, Zands y Dolatabadi (2004), evaluó la administración de la Terapia EMDR o de la Terapia Cognitivo Conductual (TCC), a 14 niñas iraníes de edades entre los 12 y 13 años, quienes fueron abusadas sexualmente.

A pesar de que las niñas reportaban síntomas de trauma y el profesor reportó problemas de conducta, no se realizó ninguna evaluación diagnóstica. Las pacientes pudieron recibir hasta 12 sesiones de tratamiento. Hubo un mínimo de 10 sesiones para la TCC pero no para la Terapia EMDR, resultando ésta última significativamente más eficaz que la TCC. Se administraron medidas de auto-reporte, reporte de los padres de familia y reporte de los maestros, antes de recibir el tratamiento y dos semanas después del tratamiento. No existió diferencia significativa entre los tratamientos. Tanto la Terapia EMDR como la TCC, produjeron efectos de gran magnitud en los resultados de la sintomatología postraumática y efectos de mediana magnitud en el decremento de los problemas conductuales en clase.

Wadda, Zaharim y Alqashan (2010), evaluaron la prevalencia de los síntomas del TEPT en niños que emigraron a Malasia huyendo de la guerra en Irak, y encontraron que el $68.5 \%$ presentaban dicha sintomatología. Los padres de 12 de estos niños (de edades entre los 7 y 12 años) accedieron a que sus hijos recibieran 12 sesiones de terapia EMDR. En la medición realizada antes del tratamiento (pre-tratamiento), no se encontraron diferencias estadísticamente significativas en los puntajes de la evaluación del TEPT entre los dos grupos de niños (con tratamiento y sin tratamiento), sin embargo, en la medición post-tratamiento, los puntajes del grupo que recibió Terapia EMDR se redujeron significativamente.

\section{El Protocolo Grupal e Integrativo con EMDR}

El Protocolo Grupal e Integrativo con EMDR (EMDRIGTP, por sus siglas en inglés), fue desarrollado por miembros de la Asociación Mexicana para Ayuda Mental en Crisis (AMAMECRISIS), para enfrentar la enorme necesidad de servicios de salud mental después de que el Huracán Paulina devastara las costas del oeste de México en el año de 1997.

Este protocolo también es conocido como el Protocolo Grupal del Abrazo de la Mariposa, el Protocolo Grupal de EMDR y el Protocolo Grupal de EMDR para niños. Para conocer la versión detallada del protocolo consulte Artigas, Jarero, Alcalá \& López Cano (2009).
Este protocolo ha sido utilizado en numerosos sitios alrededor del mundo, en su formato original o con adaptaciones para adecuarse a las circunstancias (Gelbach \& Davis, 2007; Maxfield, 2008). Reportes de casos y estudios de campo han documentado su efectividad con niños y adultos después de desastres naturales o provocados por el hombre, durante trauma de guerra en curso y crisis geopolíticas en curso (Adúriz, Knopfler, \& Bluthgen, 2009; Jarero \& Artigas 2009; Jarero \& Artigas, 2010; Jarero, Artigas, \& Hartung, 2006; Jarero, Artigas, Mauer, López Cano, \& Alcalá, 1999; Jarero, Artigas, \& Montero, 2008; Zaghrout-Hodali, Alissa, \& Dodgson, 2008).

Una nueva aplicación de este protocolo para trauma interpersonal, fue probada en la República Democrática del Congo, en donde un estudio de campo mostró que después de dos sesiones con el Protocolo Grupal e Integrativo con EMDR, las 50 adultas que habían sido víctimas de violación, reportaron el cese de los síntomas de TEPT y del dolor en la parte baja de su espalda (Allon, citado por Shapiro, 2011).

\section{La Organización Inocencia en Peligro}

Inocencia en Peligro (Innocence un Danger, IID, por sus siglas en inglés; 2011), es un movimiento mundial para la protección de los niños en contra de la violencia y la explotación sexual. Es una organización no lucrativa y no gubernamental creada por un grupo de civiles el 15 de abril de 1999. Su objetivo es implementar el plan de acción de la Junta de Expertos de la UNESCO, convocada en enero de 1999 para tratar el abuso infantil, la pornografía infantil y la pedofilia en internet. Los comités de trabajo de IID se enfocan en aumentar la conciencia pública, a través de los medios, sobre el creciente problema de la criminalidad relacionada a la pedofilia y de dar apoyo directo a niños de los 0 a los 18 años que han sufrido abuso, a través de procedimientos terapéuticos y jurídicos (IID, 2011).

Inocencia en Peligro (IID) opera en 29 países por todo el mundo con socios que comparten los mismos objetivos. Reúne militantes que son especialistas en internet, abogados, tomadores de decisiones políticas, empresarios, medios de comunicación y grupos de acción nacional. Tiene agencias en Francia, Suiza, Alemania, Estados Unidos, Inglaterra y Colombia. Cada agencia funciona como una asociación en su país y es independiente económicamente de las otras.

Desde 2008 Inocencia en Peligro-Colombia, inició sus operaciones en la ciudad de Cali, una ciudad ubicada en el suroeste del país. Su misión humanitaria, es proporcionar apoyo y tratamiento psicológico 
a niños y niñas víctimas de violencia y abogar por la protección de todos los niños, mediante la educación de la sociedad colombiana acerca del maltrato infantil en todas sus formas. IID-Colombia pretende ser una organización especializada en la atención y prevención de traumas y del estrés postraumático generado por violencia, particularmente sexual hacia niños y adolescentes. Se esfuerza por incluir a las familias y en desarrollar redes de apoyo que ayuden en este proceso.

Antes de este estudio y desde su creación, IID Colombia había llevado a cabo tres campamentos de recuperación del trauma. Cada campamento fue de 7 días, durante los cuales los niños y niñas pernoctaron o en dormitorios en la Ciudad de Cali o con sus familias en diferentes ciudades de la región. En los 3 campamentos se administró tratamiento grupal con terapia EMDR a un total 70 niños y niñas (14 en el primer campamento, 24 en el segundo y 32 en el tercero) con edades entre los 9 y 14 años, quienes habían sufrido diversos tipos de abuso. El tratamiento incluyó actividades de grupo y Terapia EMDR. Las actividades se enfocaron principalmente en la expresión corporal y verbal, en contactar con sus emociones y en trabajar su potencial creativo a través del arte. Durante los tres primeros campamentos, se implementaron y probaron los procedimientos, mismos a los que se les fueron haciendo modificaciones para optimizar la experiencia de los niños. Los resultados promisorios de esos campamentos alentaron a los autores a realizar un cuarto campamento como un estudio de investigación.

\section{Método}

\section{Participantes}

Al presente campamento de recuperación del trauma, asistieron 34 niños y niñas (18 niños y 16 niñas) con edades entre los 9 y los 14 años. Todos habían sido víctimas de violencia interpersonal severa (e.g., violación, abuso sexual, violencia física y emocional, negligencia, abandono).

La mayoría $(\mathrm{n}=32)$ habían sido víctimas de violación o abuso sexual. Un grupo $(\mathrm{n}=19,11$ niños y 8 niñas) provenía de una institución acreditada por el Instituto Colombiano del Bienestar Familiar (ICBF). Estos niños habían vivido en la calle o habían sido separados de sus familias por su conducta problemática. El otro grupo ( $n=15,7$ niños y 8 niñas), vivían con sus familias y habían sido víctimas de violación, abuso sexual y violencia física y emocional. Ninguno de los niños había recibido tratamiento especializado en trauma previo al campamento.

\section{Procedimiento}

El campamento de recuperación (CRT) del trama se llevó a cabo del 1 al 7 de diciembre de 2011. La investigación se realizó en 8 etapas:

Etapa 1: Previo al inicio del CRT, los psicólogos de la institución acreditada por ICBF, se reunieron individualmente con los niños y un miembro de la familia o de la institución, para elaborar la historia clínica y para escoger con los menores la memoria traumática que se reprocesaría durante el campamento.

Etapa 2: Antes del inicio del CRT, dos de los autores (SRL y JG) llevaron a cabo un retiro de un día para todo el equipo adulto (psicólogos, trabajadores sociales y educadores), con el objetivo de prepararlos para el trabajo que se realizaría con los niños durante el campamento.

Etapa 3: Durante el CRT (del 1 al 7 de diciembre, 2011), los niños y niñas recibieron un tratamiento para el trauma, que consistió en una aproximación terapéutica multi-componente y en fases. El 5 de diciembre se realizó la evaluación pre-tratamiento con Terapia EMDR, y el tratamiento grupal con terapia EMDR, fue administrado los días 5 y 6 de diciembre del 2011. Se realizaron los planes de dar terapia individual de EMDR a 26 niños cuya puntuación de Unidades Subjetivas de Perturbación (SUD, por sus siglas en inglés) no llegó a cero durante la intervención grupal.

Etapa 4: Los días 6 y 7 de diciembre del 2011, durante el CRT, fue administrada la terapia individual de EMDR a 6 niños (de los 11 que vivían en instituciones fuera de la Ciudad de Cali), cuyas puntuaciones de SUD para la memoria traumática elegida como blanco (diana), no alcanzaron 0 durante la intervención grupal. Dos niños recibieron una sesión individual de EMDR y 4 niños recibieron 2 sesiones.

Etapa 5: Después del CRT, entre los días 12 y 16 de diciembre de 2011, se administró la terapia individual de EMDR a 20 niños, cuyas puntuaciones de SUD no alcanzaron 0 durante la intervención grupal y quienes vivían en Cali. La mayoría de los niños ( $\mathrm{n}=18$ ), necesitaron únicamente una sesión individual y solo dos niños requirieron dos sesiones individuales. Durante esta fase, se realizó una intervención psicoeducativa familiar con 7 de los 15 niños que vivían en su casa y no en institución.

Etapa 6: Del 19 al 23 de diciembre del 2011, se aplicó la evaluación post-tratamiento.

Etapa 7: Del 27 de enero al 3 de febrero del 2011, los menores participaron en las "Jornadas de Arte y $\mathrm{Paz}$ Interna”. Los niños que residían en Cali $(\mathrm{n}=23$, niños de institución y niños de familia) asistieron el 
primer día, mientras que, los niños que provenían de alguna institución ubicada fuera de la Ciudad de Cali $(\mathrm{n}=11)$ asistieron el segundo día.

Estas jornadas consistieron en un día de actividades recreativas, artísticas y de Atención Plena (mindfulness). Su objetivo fue que los niños continuarán el proceso de sanación interna y de recuperación psicológica que iniciaron en el campamento: practicar el contacto consigo mismos, estar abiertos a los otros y socializar de manera empática.

Etapa 8: Del 8 al 10 de febrero del 2012, se aplicó la evaluación de seguimiento.

\section{Medidas}

\section{Aplicación de Escalas}

Se aplicaron la escala Short PTSD Rating Interview (SPRINT; Connor \& Davidson, 2001; Vaishnavi, Payne, Connor, \& Davidson, 2006) y la Child's Reaction to Traumatic Events Scale (CRTES; Jones, 1997) a los niños y niñas durante el CRT (Etapa 3), antes de administrar el Protocolo Grupal e Integrativo con EMDR y la terapia individual de EMDR. Estas medidas también se aplicaron en el post-tratamiento (Etapa 6) y en el seguimiento (Etapa 8). La aplicación fue realizada por psicólogos clínicos con experiencia en el Protocolo Grupal e Integrativo con EMDR. Las medidas de SUD (Shapiro, 2001) fueron tomadas durante la administración del Protocolo Grupal e individual, para monitorear el progreso del tratamiento durante la sesión, como se describe más adelante.

\section{La Escala Short PTSD Rating Interview}

La Escala Short PTSD Rating Interview (SPRINT, por sus siglas en inglés; Connor \& Davidson, 2001; Vaishnavi et al., 2006), es un cuestionario de autoevaluación de 8 reactivos, con sólidas propiedades psicométricas; que puede servir como una medida confiable, válida y homogénea, de la severidad del TEPT y del mejoramiento global; así como una medida de la perturbación somática, afrontamiento al estrés y del deterioro social, familiar y laboral.

Cada reactivo está clasificado en una escala de 5 puntos: 0 (para nada), 1 (un poco), 2 (moderadamente), 3 (bastante), y 4 (mucho). Las puntuaciones entre 18 y 32 , corresponden a síntomas marcados o severos de TEPT. De 11 a 17, corresponden a síntomas moderados. De 7 a 10, a síntomas leves, y las puntuaciones de 6 o menos, indican que no hay sintomatología o que ésta es mínima.

El SPRINT también contiene dos reactivos adicionales para medir la mejoría global, de acuerdo a un porcentaje de cambio y a una clasificación de la severidad. Esta escala fue traducida de inglés al español y de español al inglés, revisada, autorizada por uno de sus autores y adaptada a un lenguaje adecuado para niños.

El SPRINT se desempeña de forma similar a la Clinician-Administered PTSD Scale (CAPS), en la evaluación de las agrupaciones de síntomas del TEPT y en el total de la puntuación. Puede ser empleado como un instrumento diagnóstico (Vaishnavi et al., 2006). Se encontró que en el SPRINT, una puntuación de corte de 14 o más, tenía un $95 \%$ de sensibilidad para detectar el TEPT y un $96 \%$ de especificidad para descartarlo, con una precisión global de asignación correcta del 96\% (Connor \& Davidson, 2001).

\section{La Child's Reaction to Traumatic Events Scale}

La Child's Reaction to Traumatic Events Scale (CRTES, por sus siglas en inglés; Jones, 1997) se derivó de la Escala del Impacto de Eventos (Impact of Events Scale; Horowitz, Wilner, \& Álvarez, 1979). Es una escala de auto-reporte de 15 reactivos, diseñada para evaluar las respuestas psicológicas a eventos estresantes de la vida. Las respuestas se clasifican en escala Likert: 0 (nunca), 1 (raramente), 3 (algunas veces) y 5 (frecuentemente). Además del puntaje total, el CRTES proporciona puntajes para dos sub-escalas: intrusión y evitación.

Los puntajes menores de 9 son clasificados como baja perturbación, entre 9 y 18 se considera perturbación moderada y de 19 o más, como alta perturbación. A pesar de ser una evaluación de auto-reporte, los adultos capacitados para apoyar a los niños, a quienes se les denominó el Equipo de Protección Emocional (EPE), leyeron las preguntas en voz alta a los niños más pequeños. Sus respuestas fueron grabadas por el EPE. Esta evaluación se aplicó a los niños antes del tratamiento (pre-tratamiento), una semana después del tratamiento (post-tratamiento) y en el seguimiento tres meses después.

\section{Escala de Unidades Subjetivas de Perturbación}

Se utilizó una modificación de la Escala de Unidades Subjetivas de Perturbación (SUD, por sus siglas en inglés; Shapiro, 2001; Wolpe, 1958). En lugar de solicitar a los niños y niñas que simplemente reportaran su nivel de perturbación, se les mostraron dibujos de caras que representaban diferentes niveles de emociones negativas (de 0 a 10 , en donde 0 no mostraba ninguna perturbación y 10 mostraba perturbación severa), y se les pidió que seleccionaran la que mejor representara su emoción y que escribieran el número correspondiente en el dibujo. 
Para realizar esta actividad, los niños fueron apoyados por miembros del Equipo de Protección Emocional. Las puntuaciones de SUD son una parte integral del tratamiento con la Terapia EMDR (Shapiro, 2001), y su empleo ha sido demostrado en estudios de Terapia EMDR con adultos con trauma psicológico. Por ejemplo, se ha mostrado que la escala SUD tiene buena concordancia con medidas fisiológicas autonómicas de ansiedad pre y post-tratamiento (e.g., Wilson, Silver, Covi, \& Foster, 1996). La disminución en la excitación fisiológica y la relajación, se relacionaron con un decremento en las puntuaciones de SUD al final de una sesión (Sack, Lempa, Steinmetz, Lamprecht, \& Hofmann, 2008). Asimismo, las puntuaciones de SUD fueron correlacionadas significativamente con una mejoría post-tratamiento medida por terapeutas (Kim, Bae, \& Park, 2008).

\section{Tratamiento}

Taller Pre-campamento para el Equipo que administró el tratamiento.

Antes de que iniciara este campamento de recuperación del trauma y el estudio de investigación, dos de los autores (SRL y JG) llevaron a cabo un retiro para capacitar a todo el equipo adulto que participaría en el campamento (psicólogos, trabajadores sociales, artistas, educadores). Se les brindó educación sobre el tratamiento del trauma, y sobre los aspectos teóricos de la Terapia EMDR. Se les explicó que en el tratamiento de memorias traumáticas complejas, la Terapia EMDR es un componente de un plan integral de tratamiento (Tinker \& Wilson, 1999).

La capacitación también se centró en la importancia de reducir la sobre-excitación del sistema nervioso simpático. Se resaltó el valor de las actividades que se llevarían a cabo durante el campamento, ya que éstas fueron diseñadas para facilitar una experiencia de seguridad y estabilidad emocional (Courtois \& Ford, 2009).

Se puso especial atención en informar a los miembros del Equipo de Protección Emocional, sobre la importancia de tener siempre una presencia profundamente respetuosa y amorosa en los momentos emocionalmente difíciles (Jarero et al., 2008). También se les explicó que este tipo de presencia, podría incrementar las redes de memoria de información positiva, convirtiéndose en un recurso a futuro para los niños y las niñas.

En el taller también se dio información acerca del tratamiento del trauma, y se revisaron las estrategias de focalización y regulación de las emociones, así como de la práctica de la Atención Plena (ServanSchreiber, 2003).

\section{Panorama General del Tratamiento}

Durante el campamento se llevó a cabo un abordaje de tratamiento del trauma multi-componente y en fases. Esta aproximación para abordar el estrés traumático complejo fue recomendada por Courtois y Ford (2009). La primera fase de tratamiento se enfoca en la seguridad del paciente, en la estabilización de síntomas y en el mejoramiento de sus competencias básicas de vida. La segunda fase, incluye la exploración de las memorias traumáticas, reduciendo primero la perturbación emocional aguda causada por dichas memorias, y posteriormente re-evaluando su significado para integrarlas en una identidad positiva y coherente.

Los estudios empíricos que han incluido solo personas con historias de trauma complejo, han encontrado que el procesamiento de las memorias traumáticas es razonablemente bien tolerado y beneficioso, cuando se lleva a cabo de forma multi-componente (e.g., Chard, 2005).

Podría existir una ventaja al integrar la Terapia EMDR con otros tratamientos, cuando también se necesite abordar trastornos comórbidos o problemas sociales, que puedan influir en la respuesta al tratamiento (Fleming, 2012).

Tufnell (2005) concluye que la Terapia EMDR, cuando se utiliza en combinación con otros tratamientos, puede ser apropiada para niños y adolescentes con problemas de salud mental comórbidos.

En el presente estudio, la primera fase de la terapia para el tratamiento del trauma, incluyó una gama de actividades para desarrollar estabilización emocional y competencias de vida, en el contexto de un campamento terapéutico de recuperación del trauma. Esta investigación examinó la efectividad de la segunda fase del tratamiento del trauma, utilizando una combinación de terapia EMDR grupal e individual.

\section{Primera Fase del Tratamiento del Trauma}

Es importante señalar que la primera fase del tratamiento del trauma, corresponde a las Fases 1 y 2 de los procedimientos estándar de la Terapia EMDR y del Protocolo Grupal e Integrativo con EMDR. Esta fase incluyó la sesión de toma de la historia, la cual fue llevada a cabo por los psicólogos de la institución acreditada antes del campamento, y todas las experiencias, desde el día en que los niños y niñas llegaron, el 1 de diciembre, hasta el día en que dejaron el campamento, el 7 de diciembre. 
Las actividades del campamento incluyeron lo siguiente:

Por la mañana, las niñas y los niños se despertaban temprano para practicar gimnasia suave y hatha yoga. El propósito de estas actividades, fue apoyar el proceso de salud mental (Patanjali, 1991). La práctica de yoga ha sido empleada como una intervención para tratar el estrés traumático, ya que facilita el incremento de un estado de ánimo positivo, la aceptación y una actitud de paz, a través del cuidado del cuerpo y el control de la respiración (Van der Kolk, 2012).

Durante cada día, los menores se involucraron en varias actividades y talleres. Por ejemplo, un día visitaron el Museo del Oro en Cali, Colombia, como una experiencia cultural y de participación en la vida de la ciudad. Cada noche, antes de ir a dormir, los niños y las niñas tenían una fiesta con historias, relajación con sonidos suaves y música infantil.

1. El arte en diferentes expresiones

Se llevaron a cabo varios talleres de arte guiados por artistas profesionales para ayudar a los niños a contactar con su potencial creativo:

(a) El taller de pintura "Recobrando al niño/ niña que soy", tuvo como propósito que los niños encontraran a "su niño interior", que está más allá del dolor y del sufrimiento, y de esta manera, experimentar seguridad y confianza (Tafurt, comunicación personal, julio 3, 2011);

(b) Se realizaron dos talleres de música: en el taller "Acercándonos a la música a través del ritmo", los niños se dividieron en grupos para descubrir el concepto de ritmo y crear una pieza rítmica colectiva. En el taller "La música y mis sensaciones, yo creo una historia”, cada niño escribió una historia inspirada en lo que experimentaron con la música (alegría, tristeza, ternura, misterio, etc.);

(c) En el taller de escultura, los niños descubrieron el barro como material, y después de escuchar una historia con animales como protagonistas, cada niño modeló a los animales que más le gustaron, los pintaron y decoraron;

(d) En el taller de teatro, los niños presenciaron la escenificación: "El perro amigo del EMDR" (Meignant, 2007), con el objetivo de comprender la utilidad de las estimulaciones bilaterales. Éste taller se realizó el mismo día que se aplicó el Protocolo Grupal e Integrativo con EMDR, pero antes del mismo.

\section{Actividades Físicas}

Los niños y niñas se involucraron en varias actividades deportivas y recreativas, que tuvieron como propósito terapéutico alcanzar los bien conocidos beneficios del ejercicio físico, mejorar las habilidades sociales y ayudarles a reconciliarse con sus cuerpos y con el mundo que los rodea (Binswanger, 1971).

3. La práctica de la regulación emocional y la armonización

El objetivo de estas actividades fue que los niños y niñas regularan sus percepciones, pensamientos, emociones y conductas para generar armonía interior. Estas prácticas se llevaron a cabo durante las siguientes actividades:

(a) Historias y relajación siguiendo las recomendaciones de Lovett (1999). Al inicio, la historia presenta algo positivo con el fin de atraer la atención de los niños, posteriormente se describe un evento traumático y los síntomas relacionados, y al final, la historia concluye con la resolución del trauma y creencias positivas;

(b) Los niños aprendieron la práctica de la Atención Plena (Mindfulness; Williams, Teasdale, Segal, \& Kabat-Zinn, 2007), con el fin de aprender a observar la experiencia directa de su cuerpo, y para desarrollar una actitud compasiva hacia ellos mismos (Nhat-Hanh, 1974). También realizaron el ejercicio de coherencia cardíaca (Deglon, 2006);

(c) En el taller de valores humanos, los niños y niñas usaron su imaginación y creencias positivas, para visualizar un futuro lleno de posibilidades realistas e integradas con valores humanos profundos;

(d) La orientación espiritual se enseño no desde una visión religiosa, sino alentando a los niños y niñas a cuidar de sí mismos y del mundo que les rodea;

(e) Se les dieron masajes biodinámicos psicoterapéuticos a los niños, para favorecer la relajación y contribuir a que tuvieran sentimientos de seguridad dentro de su propio cuerpo (Boyesen, 1985).

4. Preparación específica para la Terapia EMDR Para prepararse para la Terapia EMDR, los niños realizaron una actividad teatral llamada "El delfín y la muñeca Lupita", en donde aprendieron la técnica de la respiración abdominal y el reconocimiento de emociones. Ambas actividades se describen en el Protocolo Grupal e Integrativo con EMDR para niños (Jarero et al., 2008). 
Las actividades de "crear su lugar seguro" y "normalizar las reacciones" (que tienen como propósito que los niños reconozcan, validen y normalicen los signos y síntomas del estrés postraumático), se realizaron como se describe en el Protocolo Grupal e Integrativo con EMDR para niños.

Miembros del Equipo de Protección Emocional, apoyaron a los niños en el aprendizaje y la práctica del Abrazo de la Mariposa (Artigas, 2011), y completaron el ejercicio de "las caritas de las emociones", una herramienta empleada en el Protocolo Grupal e Integrativo con EMDR, para enseñar a los niños a observar y comunicar sus niveles de perturbación emocional y las puntuaciones de SUD.

\section{Segunda Fase del Tratamiento para el Trauma}

Es importante hacer notar que la segunda fase de tratamiento del trauma, corresponde a las Fases 3 a 7 de la Terapia EMDR (Shapiro, 2001). En el presente estudio se empleó el Protocolo Grupal e Integrativo con EMDR con los 34 participantes, y la terapia individual de EMDR con 26 participantes.

El Protocolo Grupal e Integrativo con EMDR estuvo incorporado dentro de las actividades del campamento. Las sesiones individuales de EMDR se administraron el último día del campamento, a 6 participantes que vivían fuera de la Ciudad de Cali, y para los otros 20 niños y niñas, se dio después de haber terminado el campamento.

Estas terapias se administraron de acuerdo a los protocolos estándar (Artigas et al., 2009; Shapiro, 2001) por tres terapeutas certificados en Terapia EMDR, quienes estaban capacitados y tenían experiencia trabajando con niños y poblaciones vulnerables.

El Protocolo Grupal e Integrativo con EMDR. La terapia grupal se administró a los 34 participantes en un solo grupo, y el tratamiento tuvo una duración de 6 horas. Se administró en tres ocasiones, a lo largo de tres días. En el primer día los menores completaron la fase preparatoria del Protocolo Grupal e Integrativo con EMDR, en el segundo día, hicieron las fases de 3 a 7 (primera medición del SUD); y en el tercer día volvieron a hacer las fases 3 a 7 (segunda y última medida de SUD).

Nota: Para no hacer daño (re-traumatizar) a los participantes, solo personas entrenadas formalmente en terapia EMDR y en el Protocolo Grupal e Integrativo con EMDR, pueden aplicar los protocolos.

Terapia Individual de EMDR. Las sesiones de terapia individual tuvieron una duración de 60 minutos. La memoria a trabajar fue la misma que se utilizó para las sesiones grupales. Se utilizaron dibujos durante la sesión, y también se les pidió a los niños que identificaran una creencia negativa. El tratamiento se llevó a cabo siguiendo los procedimientos estándar de la Terapia EMDR.

\section{Resultados}

Los fuertes efectos del tratamiento con el Protocolo Grupal e Integrativo con EMDR, resultan evidentes al observar el decremento de las puntuaciones de SUD (ver Figura1). Los niveles de perturbación subjetiva en los niños y niñas disminuyeron durante la primera sesión, se mantuvieron, y redujeron aún más durante la segunda sesión.

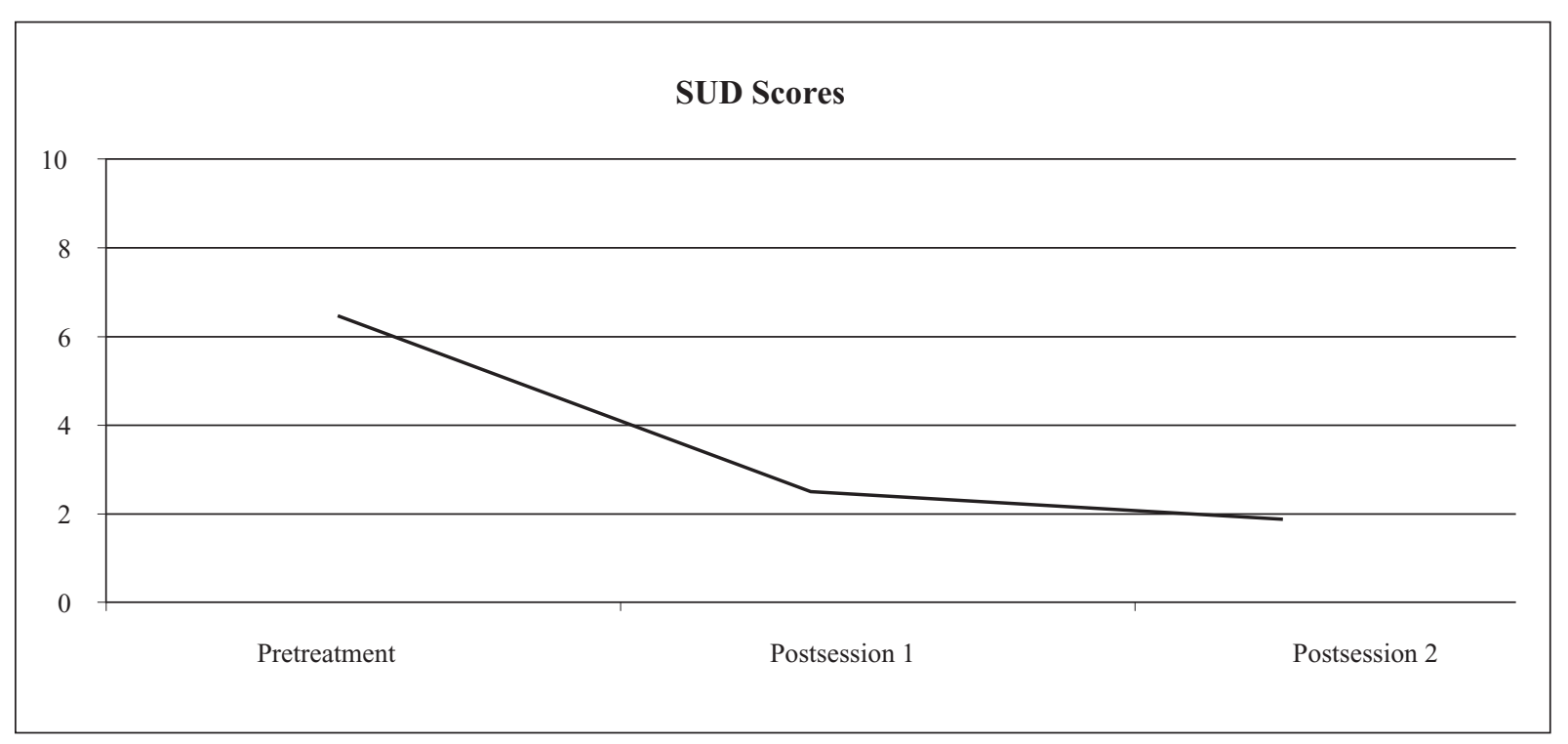

FIGURA 1. Cambios en las puntuaciones de las Unidades Subjetivas de Perturbación (SUD) durante las sesiones del Protocolo Grupal e Integrativo con EMDR. 1. Pre-tratamiento, 2. Post-sesión 1, 3. Post-sesión 2. 
TABLA 1. Puntajes de la Media y Desviación Estándar de la Escala SPRINT.

\begin{tabular}{lccc}
\hline \multicolumn{4}{c}{ SPRINT } \\
Estadísticas Descriptivas \\
\hline POBLACIÓN & Media & Desviación Std. & N \\
\hline Pre-tratamiento & & & \\
INSTITUCIÓN & 18.95 & 6.786 & 19 \\
FAMILIA & 16.73 & 5.824 & 15 \\
Total & 17.97 & 6.384 & 34 \\
Post-tratamiento & & & \\
INSTITUCIÓN & 4.53 & 3.657 & 19 \\
FAMILIA & 4.73 & 2.963 & 15 \\
Total & 4.62 & 3.321 & 34 \\
Seguimiento & & & 19 \\
INSTITUCIÓN & 2.11 & 2.726 & 34 \\
FAMILIA & 1.00 & 1.254 & \\
Total & 1.62 & 2.243 & \\
\hline
\end{tabular}

Los efectos del programa completo fueron medidos con las escalas CRTES y SPRINT (Connor $\&$ Davidson, 2001; Vaishnavi et al., 2006), las cuales se administraron a los dos grupos de niños y niñas (provenientes de familia o de institución) en tres ocasiones: pre-tratamiento, pos-tratamiento y seguimiento.

Las Tablas 1 y 2, muestra las medias y desviaciones estándar obtenidas en ambos grupos para cada instrumento que se aplicó a lo largo del tiempo.

\section{Comparación de los efectos del Tratamiento en Dos Grupos Diferentes}

Se aplicó un Modelo General Lineal, con el objetivo de conocer si las diferencias encontradas, se debían a la condición de los participantes (si provenían de familia o institución). Los puntajes de ambos instrumentos (CRTES y SPRINT) se compararon para las medidas repetidas (pre-tratamiento, post-tratamiento y seguimiento), en cada uno de los grupos (institución y familia). Los resultados no mostraron efectos significativos para la interacción entre puntajes de instrumentos y el tipo de grupo. Esto indica que los resultados del tratamiento siguen un patrón similar en ambos grupos (institución y familia).

El Análisis de Varianza (ANOVA), muestra un efecto significativo en el tiempo para los dos instrumentos, observándose que todos los participantes $(\mathrm{N}=34)$ tuvieron una mejoría en el CRTES $(F[1,33]=520.26$, $p<.001)$ y en el SPRINT $(F[1,33]=259.27, p<.001)$.

Todos los puntajes del SPRINT en el seguimiento indican síntomas de TEPT mínimos o muy leves
TABLA 2. Puntajes de la Media y Desviación Estándar de la Escala CRTES.

\begin{tabular}{lccc}
\hline \multicolumn{4}{c}{ CRTES } \\
Estadísticas Descriptivas \\
\hline POBLACIÓN & Media & Desviación Std. & $\mathrm{N}$ \\
\hline Pre-tratamiento & & & \\
$\quad$ INSTITUCIÓN & 35.79 & 9.157 & 19 \\
FAMILIA & 35.13 & 7.945 & 15 \\
Total & 35.50 & 8.522 & 34 \\
Post-tratamiento & & & \\
INSTITUCIÓN & 9.84 & 6.283 & 19 \\
FAMILIA & 5.80 & 3.489 & 15 \\
$\quad$ Total & 8.06 & 5.554 & 34 \\
Seguimiento & & & \\
INSTITUCIÓN & 4.42 & 3.641 & 19 \\
FAMILIA & 2.33 & 2.289 & 15 \\
Total & 3.50 & 3.250 & 34 \\
\hline
\end{tabular}

(Figura 2). Además, todos los puntajes del CRTES en el seguimiento indicaron baja perturbación (Figura 3).

Con el fin de evaluar los cambios en todo el grupo, y en cada grupo por separado, se llevaron a cabo pruebas t post hoc planeadas. Estas mostraron una reducción significativa en los síntomas para el CRTES $(t[33]=$ $15.49, p<.001)$ y para el SPRINT $(t[33]=19.87, p<$ .001; ver Tablas 1 y 2, así como Figuras 2 y 3.

Estos resultados confirman el efecto del programa y que éste se mantuvo durante el tiempo. Finalmente, la rápida variación en las puntuaciones de SUD durante las sesiones de procesamiento (Figura 1) es consistente con resultados anteriores.

Mejoria Subjetiva Global. El SPRINT contiene dos reactivos para medir la mejoría global, uno evalúa el porcentaje de cambio y el otro el nivel de severidad. Ítem 1: ¿Cuánto mejor se ha sentido desde que inicio el tratamiento? En un porcentaje del 0 al 100. Ítem 2: ¿Cuánto han mejorado los síntomas mencionados arriba desde que inició el tratamiento?: 1(empeorado), 2 (sin cambio), 3 (mínimamente), 4 (mucho), 5 (muchísimo).

Para el grupo total de participantes, la media obtenida en el seguimiento fue de $95 \%$ en el reactivo 1 ; mientras que para el reactivo 2 fue de: Mucha mejoría.

El Seguimiento en las Jornadas de Arte y Paz Interior. Varios niños y niñas expresaron que deseaban encontrarse con sus compañeros para la sesión de seguimiento llamada "Jornadas de Arte y Paz Interior". Durante las Jornadas (del 27 de enero al 3 de febrero, 2012), las observaciones clínicas de los autores fueron: 


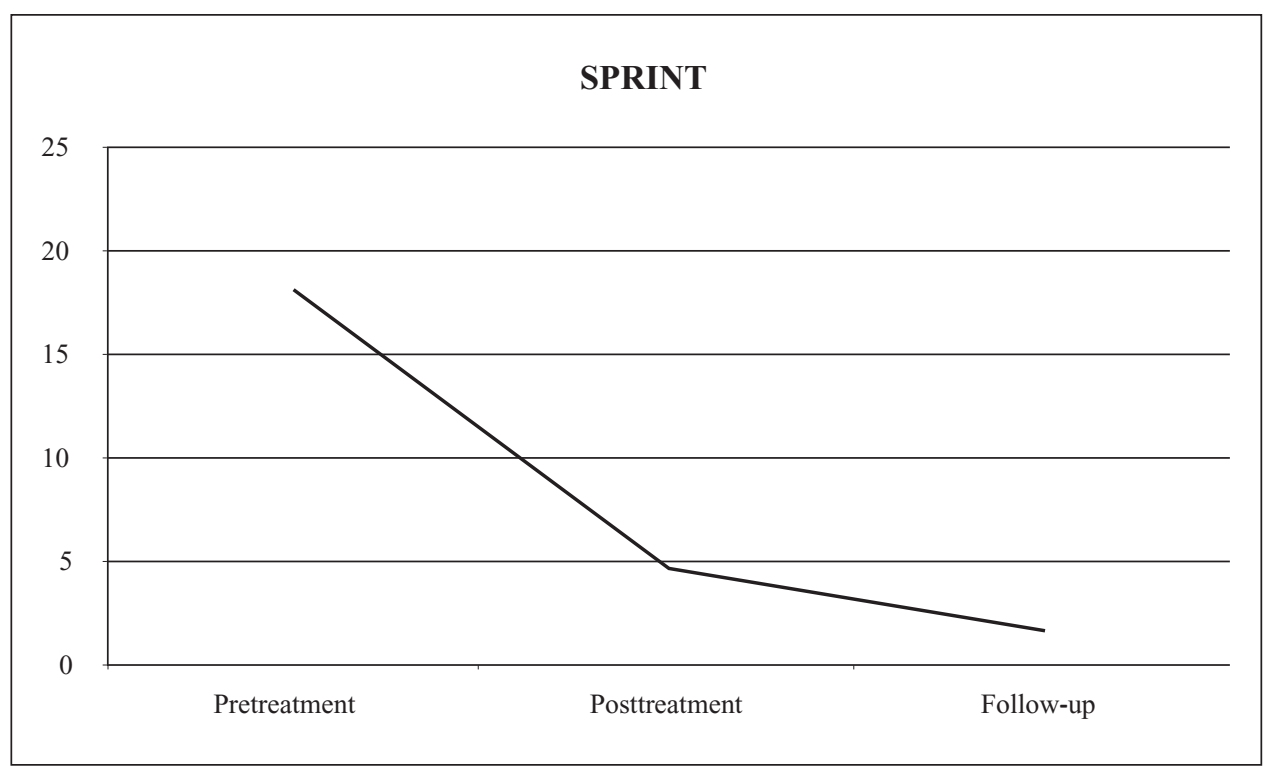

FIGURA 2. Cambios en los Puntajes la Escala Short PTSD Rating Interview (SPRINT). 1. Pre-tratamiento, 2. Post-tratamiento, 3. Seguimiento.

que los niños y niñas tenían un nuevo sentido del placer y una mayor capacidad para disfrutar y divertirse.

\section{Discusión}

Los resultados obtenidos muestran efectos significativos del tratamiento con Terapia EMDR en todos los menores, independientemente de si éstos vivían con su familia o provenían de una institución. Los efectos del tratamiento se mantuvieron a lo largo del tiempo, como lo muestran los resultados de las mediciones realizadas en el seguimiento.

\section{Tratamiento Multimodal}

Los niños y niñas recibieron un tratamiento multimodal, en el que la primera fase del tratamiento terapéutico se realizó en el contexto de un campamento de recuperación del trauma psicológico. Como se ha descrito previamente, las actividades fueron diseñadas para incrementar la conciencia de sí mismos y la auto-aceptación.

Los participantes aprendieron estrategias focalizadas en las emociones y para la regulación de las mismas, así como la práctica de la Atención Plena (mindfulness). Estas prácticas han sido evaluadas por expertos en

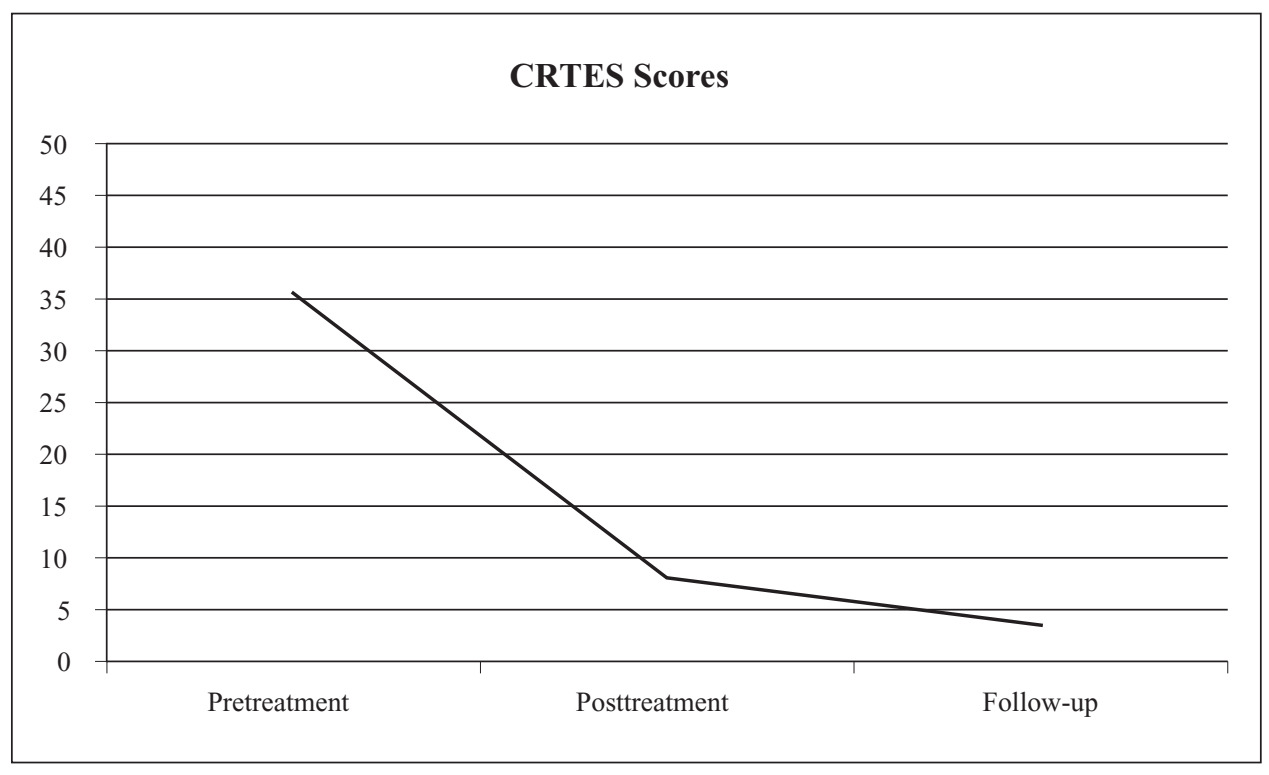

FIGURA 3. Cambios en los puntajes de la Child's Reaction To Traumatic Events Scale (CRTES) después del tratamiento. 1. Pre-tratamiento, 2. Post-tratamiento, 3. Seguimiento. 
trauma como de primera y segunda línea para la Fase 1 del tratamiento del TEPT (Cloitre et al., 2011).

La terapia multimodal que se aplicó en este estudio, abordó muchas de las diversas necesidades terapéuticas de los niños. Este programa con duración de una semana, parece ser suficiente, para que los niños y niñas pre-adolescentes con trauma complejo, experimenten cambios significativos, que les permitan seguir adelante en sus vidas, con nuevos sentimientos de fortaleza y júbilo.

Desde la perspectiva de la Teoría del Procesamiento de la Información (Shapiro, 2001), los autores consideran que uno de los beneficios clave de las diversas actividades que se realizaron, fue la creación y fortalecimiento de redes de memoria positivas. Estas redes estuvieron accesibles a los niños y niñas durante el procesamiento de sus memorias traumáticas con EMDR. Se tiene la hipótesis de que esto permitió a los niños y niñas reprocesar las memorias traumáticas, sin sentirse abrumados por perturbaciones emocionales.

Este estudio arrojó resultados positivos, a favor del tratamiento multimodal de una semana, que incluyó el Protocolo Grupal e Integrativo con EMDR y una o dos sesiones individuales de Terapia EMDR. Fue un tratamiento efectivo y limitado en tiempo que además, al tratar un mayor número de niños, ofrece ventajas en términos de costos.

Parece ser una alternativa eficiente al tipo de tratamientos que se utiliza normalmente con niños, los cuales incluyen sesiones grupales e individuales que se extienden por un período de 2 o 3 meses. La investigación ha demostrado que los niños con TEPT, mejoran sustancialmente en terapias con una duración de 9 a 12 semanas, y las respuestas de la encuesta ISTSS (Cloitre, et al., 2011), sugieren un período más largo para el tratamiento de TEPT complejo.

Nosotros recomendamos que el tratamiento multimodal con el Protocolo Grupal e Integrativo con EMDR y la terapia individual de EMDR, sea considerado como una poderosa opción alterna a las aproximaciones tradicionales.

\section{La Terapia EMDR y el Protocolo Grupal e Integrativo con EMDR}

Los resultados del presente estudio, demuestran la efectividad de la combinación del Protocolo Grupal e Integrativo con EMDR y la terapia individual de EMDR, para resolver los síntomas de TEPT, en niños que han vivido violencia interpersonal severa.

Los descubrimientos son consistentes, con otros estudios que han investigado la utilización de esta aproximación en niños que han sufrido traumas. Sin embargo, la aplicación de las modalidades individual y grupal de Terapia EMDR en este estudio, es única por diferentes motivos importantes:

Los otros estudios encontraron que el Protocolo Grupal e Integrativo con EMDR, es efectivo y eficiente en el tratamiento de grupos de personas que experienciaron juntos un desastre específico. En este estudio, los eventos traumáticos, en los que se enfocaron los niños y niñas para su tratamiento con la Terapia EMDR, fueron eventos individuales. Sin embargo, el protocolo grupal fue apropiado y efectivo. El tratamiento fue administrado a los participantes con trauma complejo en tres ocasiones, en lugar de una sola administración, como ha sido común en otros estudios (ver Figura 1).

También es el primer estudio que ha medido los efectos de la combinación del Protocolo Grupal e Integrativo con EMDR y la terapia individual de EMDR. Se observó que las tres sesiones grupales, tuvieron efectos en la disminución de las puntuaciones de SUD, asociadas a la memoria traumática; y con el aumento en la sensación de dominio y confianza con la que podían enfrentar las memorias aterradoras. Solo se necesitaron una o dos sesiones de terapia individual de EMDR, para estos niños y niñas que habían vivido diversos traumas interpersonales severos alcanzaran los SUD de 0 .

Es importante hacer notar, que a pesar de que al inicio del tratamiento grupal, únicamente se eligió una memoria para ser procesada, los efectos se generalizaron a toda la red que agrupaba incidentes similares (e.g., violación, abuso sexual, violencia física y emocional, negligencia, abandono). Shapiro (2001), menciona que cuando el cliente elige un incidente a reprocesar que representa una agrupación (cluster) de memorias en particular, "los reportes clínicos han verificado que habitualmente ocurre la generalización, causando un efecto de reprocesamiento en toda la agrupación (cluster) de incidentes" (p. 207).

Los resultados de este estudio sugieren que la combinación del Protocolo Grupal e Integrativo con EMDR y la terapia individual de EMDR, puede ser una potente aproximación terapéutica, que puede ser utilizada en diferentes escenarios.

\section{Recomendaciones}

Este programa es apropiado para el contexto actual en Colombia. El cese de los conflictos y la construcción de una paz duradera, otorga la oportunidad de restablecer la salud mental de aquellos individuos víctimas del conflicto. Los autores proponen la implementación de este programa para todos los niños y 
niñas colombianos que hayan sido víctimas de violencia interpersonal severa.

A la fecha, existen pocos estudios que analicen adaptaciones $u$ otras alternativas de tratamiento diferentes a aquellas ya establecidas para tratar TEPT, específicamente en personas con historias de trauma complejo. Los autores recomiendan mayor investigación en el futuro sobre el uso de la Terapia EMDR, como parte de una aproximación multi-componente y en fases para el tratamiento del trauma.

\section{Referencias}

Adler-Tapia, R. L., \& Settle, C. S. (2008). EMDR and the art of psychotherapy with children. New York, NY: Springer.

Adúriz, M. E., Knopfler, C., \& Bluthgen, C. (2009). Helping child flood victims using group EMDR intervention in Argentina: Treatment outcome and gender differences. International Journal of Stress Management, 16(2), 138-153.

American Psychiatric Association. (2000). Diagnostic and statistical manual of mental disorders (4th ed., Text Rev.). Washington, DC: Author.

Artigas, L. (2011). Escenas detrás de las alas del abrazo de la mariposa [Behind the scenes of the butterfly hug]. Revista Iberoamericana de Psicotraumatología y Disociación, 1 (1), 1-10. Retrieved from http:/ / revibapst.com/ ARTICULO\%20LUCY\%202011.pdf

Artigas, L., Jarero, I., Alcalá, N., \& López Cano, T. (2009). The EMDR integrative group treatment protocol (IGTP). In M. Luber (Ed.), Eye movement desensitization and reprocessing (EMDR) scripted protocols: Basic and special situations (pp. 279-288). New York, NY: Springer.

Binswanger, L. (1971). Introduction à l'analyse existentielle. Paris, France: Editions de Minuit.

Bisson, J., \& Andrew, M. (2007). Psychological treatment of post-traumatic stress disorder (PTSD). Cochrane Database of Systematic Reviews, (3), CD003388. Retrieved from http: / / www2.cochrane.org/reviews/ en/ab003388.html Boyesen, G. (1985). Entre psyché et soma. Paris, France: Payot. Brewing, C. R., Andrews, B., \& Valentine, J. D. (2000). Meta-analysis of risk factors for posttraumatic stress disorder in trauma exposed adults. Journal of Consulting and Clinical Psychology, 68, 748-766.

Chard, K. M. (2005). An evaluation of cognitive processing therapy for the treatment of posttraumatic stress disorder related to childhood sexual abuse. Journal of Consulting and Clinical Psychology, 73, 965-971.

Chemtob, C. M., Nakashima, J., \& Carlson, J. G. (2002). Brief treatment for elementary school children with disaster-related posttraumatic stress disorder: A field study. Journal of Clinical Psychology, 58(1), 99-112.

Cloitre, M., Courtois, C. A., Charuvastra, A., Carapezza, R., Stolbach, B. C., \& Green, B. L. (2011). Treatment of complex PTSD: Results of the ISTSS expert clinician survey on best practices. Journal of Traumatic Stress, $24(6), 615-627$.
Cocco, N., \& Sharpe, L. (1993). An auditory variant of eye movement desensitization in a case of childhood posttraumatic stress disorder. Journal of Behavioral Therapy and Experimental Psychiatry, 24(4), 373-377.

Connor, K. M., \& Davidson, J. R. T. (2001). SPRINT: A brief global assessment of post-traumatic stress disorder. International Clinical Psychopharmacology, 16(5), 279-284.

Courtois, C. A., \& Ford, J. D. (2009). Treating complex traumatic stress disorders: An evidence-based guide. New York, NY: Guilford Press.

De Bellis, M., \& Van Dillen, T. (2005). Childhood posttraumatic stress disorder: An overview. Child and Adolescent Psychiatric Clinics of North America, 14,745-772.

Deglon, C. (2006). Les premiers pas avec la Cohérence Cardiaque. Paris, France: P. I. Conseil.

EMDR International Association. (2011, September). Update EMDRIA definition of EMDR. EMDRIA Newsletter. Retrieved from http://www.emdria.org/ associations / 12049/files / SeptemberNewsletter2011.pdf

Fernandez, I. (2007). EMDR as treatment of post-traumatic reactions: A field study on child victims of an earthquake. Educational and Child Psychology, 24(1), 65-72.

Fleming, J. (2012). The effectiveness of eye movement desensitization and reprocessing in the treatment of traumatized children and youth. Journal of EMDR Practice and Research, 6(1), 16-26.

Gelbach, R., \& Davis, K. (2007). Disaster response: EMDR and family systems therapy under communitywide stress. In F. Shapiro, F. W. Kaslow, \& L. Maxfield (Eds.), Handbook of EMDR and family therapy processes (pp. 387406). New York, NY: John Wiley \& Sons.

Greenwald, R. (1994). Applying eye movement desensitization and reprocessing (EMDR) to the treatment of traumatized children: Five case studies. Anxiety Disorders Practice Journal, 1, 83-97.

Horowitz, M., Wilner, N., \& Alvarez, W. (1979). Impact of events scale: A measure of subjective stress. Psychosomatic Medicine, 41, 209-218.

Innocence in Danger. (2011). Connaître IID. Retrieved from http:/ / innocenceendanger.org/innocence-en-danger/ organisation

Jaberghaderi, N., Greenwald, R., Rubin, A., Zands, S. O., \& Dolatabadi, S. (2004). A comparison of CBT and EMDR for sexually-abused Iranian girls. Clinical Psychology and Psychotherapy, 11(5), 358-368.

Jarero, I., \& Artigas, L. (2009). EMDR integrative group treatment protocol. Journal of EMDR Practice \& Research, 3(4), 287-288.

Jarero, I., \& Artigas, L. (2010). EMDR integrative group treatment protocol: Application with adults during ongoing geopolitical crisis. Journal of EMDR Practice and Research, 4(4), 148-155.

Jarero, I., Artigas, L., \& Hartung, J. (2006). EMDR integrative treatment protocol: A post-disaster trauma intervention for children $\&$ adults. Traumatology, 12, 121-129. 
Jarero, I., Artigas, L., Mauer, M., López Cano, T., \& Alcalá, N. (1999, November). Children's post-traumatic stress after natural disasters: Integrative treatment protocol. Poster session presented at the annual meeting of the International Society for Traumatic Stress Studies, Miami, FL.

Jarero, I., Artigas, L., \& Montero, M. (2008). The EMDR integrative group treatment protocol: Application with child victims of a mass disaster. Journal of EMDR Practice and Research, 2, 97-105.

Jones, R. (1997). Child's reaction to traumatic events scale (CRTES). In J. Wilson \& T. Keane (Eds.), Assessing psychological trauma and PTSD (2nd ed.). New York, NY: Guilford Press.

Kemp, M., Drummond, P., \& McDermott, B. (2010). A wait-list controlled pilot study of eye movement desensitization and reprocessing (EMDR) for children with post-traumatic stress disorder (PTSD) symptoms from motor vehicle accidents. Clinical Child Psychology and Psychiatry, 15(1), 5-25.

Kim, E., Bae, H., \& Park, Y. C. (2008). Validity of the subjective units of disturbance scale (SUDS) in EMDR. Journal of EMDR Practice and Research, 1, 57-62.

Lovett, J. (1999). Small wonders, healing childhood trauma with EMDR. New York, NY: The Free Press.

Maxfield, L. (2008). EMDR treatment of recent events and community disasters. Journal of EMDR Practice \& Research, 2(2), 74-78.

Meignant, I. (2007). L'EMDR de Bouba le chien. Paris, France: Editions Maignant.

Nhat Hanh, T. (1974). The miracle of being awake. Boston, MA: Beacon Press.

National Collaborating Centre for Mental Health. (2005). Post-traumatic stress disorder: The management of PTSD in adults and children in primary and secondary care. National clinical practice guideline number 26. Wiltshire, United Kingdom: Cromwell Press Limited.

Parnell, L. (1999). EMDR in the treatment of adults abused as children. New York, NY: W. W. Norton.

Patanjali, S. (1991). Yoga sutras. In J. H. Woods (Ed.), Yoga sutras de Patanjali (p. 29). Paris, France: Albin Michel.

Pecora, P. J., White, C. R., Jackson, L. J., \& Wiggins, T. (2009). Mental health of current and former recipients of foster care: A review of recent studies in the USA. Child and Family Social Work, 14, 132-146.

Ribchester, T., Yule, W., \& Duncan, A. (2010). EMDR for childhood PTSD after road traffic accidents: Attentional, memory, and attributional processes. Journal of EMDR Practice and Research, 4(4), 138-147.

Sack, M., Lempa, W., Steinmetz, A., Lamprecht, F., \& Hoffmann, A. (2008). Alteration in autonomic tone during trauma exposure using eye movement desensitization and reprocessing (EMDR)-results of a preliminary investigation. Journal of Anxiety Disorders, 22(7), 1264-1271.

Servan-Schreiber, D. (2003). Guérir, le stress, l'anxiété et la dépression sans médicaments ni psychanalyse. Paris, France: Editions Robert Laffont.
Shapiro, F. (2001). Eye movements desensitization and reprocessing. Basic principles, protocols, and procedures (2nd ed.). New York, NY: Guilford Press.

Shapiro, F. (2011, September). EMDR therapy update: Theory, research and practice. Paper presented at the EMDR International Association Conference in Anaheim, CA.

Tinker, R. H., \& Wilson, S. (1999). Through the eyes of a child, EMDR with children. New York, NY: W. W. Norton.

Tufnell, G. (2005). Eye movement desensitization and reprocessing in the treatment of pre-adolescent children with post-traumatic symptoms. Clinical Child Psychology and Psychiatry, 10(4), 587-600.

United Nations. (2006). Report of the independent expert Paulo Sérgio Pinheiro for the United Nations study on violence against children. Retrieved from http://daccess-ddsny.un.org/doc/UNDOC/GEN/N06/491 / 05 / PDF/ N0649105.pdf?OpenElement

Van der Kolk, B. A. (2002). Assessment and treatment of complex PTSD. In R. Yehuda (Ed.), Treating trauma survivors with PTSD (pp. 127-153). Washington, DC: American Psychiatric.

Van der Kolk, B. A. (2005). Developmental trauma disorder: Toward a rational diagnosis for children with complex trauma histories. Psychiatric Annals, 35, 401-408.

Van der Kolk, B. A. (2012, June). Trauma in different mental disorders. Paper presented at the 13th Conference EMDR Europe, Madrid, Spain.

Vaishnavi, S., Payne, V., Connor, K., \& Davidson, J. R. (2006). A comparison of the SPRINT and CAPS assessment scales for posttraumatic stress disorder. Depression and Anxiety, 23(7), 437-440.

Wadda, N. N., Zaharim, N. M., \& Alqashan, H. F. (2010). The use of EMDR in treatment of traumatized Iraqi children. Digest of Middle East Studies, 19(1), 26-36.

Williams, M., Teasdale, J., Segal, Z., \& Kabat-Zinn, J. (2007). Méditer pour ne pas déprimer. Paris, France: Editions Odile Jacob.

Wilson, D. L., Silver, S. M., Covi, W. G., \& Foster, S. (1996). Eye movement desensitization and reprocessing: Effectiveness and autonomic correlates. Journal of Behavior Therapy and Experimental Psychiatry, 27(3), 219-229.

Wolpe, J. (1958). Psychotherapy by reciprocal inhibition. Stanford, CA: Stanford University Press.

Zaghrout-Hodali, M., Alissa, F., \& Dodgson, P. (2008). Building resilience and dismantling fear: EMDR group protocol with children in an area of ongoing trauma. Journal of EMDR Practice \& Research, 2(2), 106-113.

Reconocimientos. Los autores desean agradecer a la Dra. Martha Givaudán su valiosa contribución en el análisis de datos de este estudio.

La correspondencia referente a este artículo, debe de ser dirigida a Ignacio Jarero, Ph.D., Ed.D. Boulevard de la Luz 771, Jardines del Pedregal, Álvaro Obregón, México City 01900. E-mail: nacho@amamecrisis.com.mx 